\title{
Identification of Factors Related to Cases of Benign Paroxysmal Positional Vertigo Refractory to Canalicular Repositioning Maneuvers and Evaluation of the Need for Magnetic Resonance Imaging in their Management: Retrospective Analysis of a Series of 176 Cases
}

\author{
Claudio Carnevale ${ }^{1}$ Guillermo Til Pérez ${ }^{1}$ Diego Arancibia Tagle ${ }^{1}$ Manuel Tomás Barberán ${ }^{1}$ \\ Pedro Sarría Echegaray ${ }^{1}$
}

${ }^{1}$ ENT Department, Hospital Universitari Son Espases, Palma de Mallorca, Islas Baleares, Spain

Int Arch Otorhinolaryngol 2019;23:196-202.
Address for correspondence Claudio Carnevale, MD, ENT Department, Hospital Universitari Son Espases, Carretera Valldemossa 79, Palma de Mallorca, Islas Baleares 07120, Spain (e-mail: claudio.carnevale.orl@gmail.com).

\begin{abstract}
Introduction Benign paroxysmal positional vertigo (BPPV) is the most common form of peripheral vertigo, and, in most cases, it presents a favorable prognosis. The treatment is based on a series of specific canalicular repositioning maneuvers that offer an efficacy close to $100 \%$. Despite this, there are cases that are refractory to treatment, with the persistence of the vertigo symptoms.

Objectives The objective of the present paper is to analyze the factors associated with an increased risk of refractory BPPV and the importance of nuclear magnetic resonance in the study of these patients.

Methods We retrospectively reviewed the cases of 176 patients diagnosed with BPPV in our center. We divided them into two groups: responders and non-responders to the treatment, and analyzed the possible risk factors associated with a higher risk of refractory vertigo. Fischer exact test was used.

Results We found 11 cases refractory to treatment; all of them underwent magnetic resonance imaging (MRI) with gadolinium according to our protocol. Of these, four had an otoneurologic background or pathology, and two other patients presented a multicanal involvement. The difference between the two groups was statistically significant $(p<0.05)$.

Conclusion Otoneurologic background and multicanal involvement were associated with

\section{Keywords}

- vertigo

- positional vertigo

- central origin a higher risk of refractory BPPV. When dealing with a BPPV with persistent symptomatology/ nystagmus or with early relapse after an initial improvement, other entities that enter into the differential diagnosis must always be considered. We consider it essential to perform an MRI with gadolinium to rule out cases of BPPV that have a central cause.
\end{abstract}

(D) Claudio Carnevale's ORCID is https://orcid.org/0000-0003-34119803.

received

March 18, 2018

accepted

June 20, 2018

published online

October 26, 2018
DOI https://doi.org/

10.1055/s-0038-1670693. ISSN 1809-9777.
Copyright $\odot 2019$ by Thieme Revinter

Publicações Ltda, Rio de Janeiro, Brazil
License terms

(c) $(1) \$$ 


\section{Introduction}

Benign paroxysmal positional vertigo (BPPV) is the most common form of peripheral vertigo, representing $\sim 25 \%$ of all vertigos of vestibular origin. An incidence of 64/100,000 inhabitants per year is estimated in the United States, with a lifetime prevalence of $2.4 \%$. However, due to its high rate of spontaneous remissions, it is very difficult to estimate its real incidence. ${ }^{1}$ It is characterized by a recurrent vertigo, which lasts for seconds, is caused by specific positions of the head in space, and is not accompanied by other otoneurologic symptoms. It usually affects patients older than 45 years of age (up to $9 \%$ of the population over 60 years old), and has a higher incidence among women, with a ratio of $2: 1^{1}$ The exact etiology of BPPV is still a matter of debate. In any case, more than $50 \%$ of the cases are idiopathic, primary BPPV; the remaining cases are of secondary BPPV. ${ }^{2}$ Among the latter, the most frequent cause is traumatic brain injury, including those cases of mild severity (18-20\%), and viral labyrinthitis (16\%). Ischemia of the anterior vestibular artery or surgery of the middle or inner ear (especially stapedectomy) are possible less frequent causes. ${ }^{3}$ Other factors associated with BPPV have also been described: Ménière disease, migraine, vertebrobasilar insufficiency, prolonged bed rest, ototoxicity, depression over the past year and cardiovascular risk factors, such as hypertension, hyperlipidemia, diabetes, coronary disease and obesity. According to the latest research, the rates of involvement of the posterior semicircular canal (PSC), the horizontal semicircular canal (HSC), and the superior semicircular canal (SSC) are 60 to $90 \%, 10$ to $25 \%$ and 1 to $2 \%$ respectively. ${ }^{4}$ Although first described by Barany in 1921 , the specific characteristics of this disorder were defined in 1952 by Dix and Hallpike. The proposed treatments range from observation (considering BPPV a self-limiting disorder) to aggressive surgical procedures, such as section of the posterior ampullary nerve or occlusion of the posterior semicircular canal. The conservative treatments, which are currently used and recommended, include canalicular repositioning maneuvers and habituation exercises. ${ }^{5,6}$ The former, described by Brandt and Daroff ${ }^{7}$ and Norré and Beckers, ${ }^{8}$ aim to increase the tolerance of the vestibular system to vertigo rather than eliminate the cause, and are used both as an initial treatment and as a treatment secondary to the failure of the repositioning maneuvers.

The repositioning maneuvers are all based on the two currently accepted etiopathogenic theories, the theory of cupulolithiasis and the theory of canalolithiasis, ${ }^{6}$ both described by Schuknecht. The Epley maneuver (EM) and the Semont maneuver are for the posterior semicircular canal; the Gufoni and Lempert maneuver, for the horizontal semicircular canal; and the Yacovino maneuver, for the superior semicircular canal. The particle replacement maneuvers are very effective, especially in the case of the posterior duct: it is estimated that they resolve 90 to $95 \%$ of the cases with an average of 1 to 3 maneuvers ${ }^{9-12}$ This resolution rate is lower for the HSC (85\%), ${ }^{11,13,14}$ and it is difficult to estimate, due to the scarce casuistry, the resolution rate for the SSC.
However, there are patients ( 3.5 to $12 \%$, depending on the series $)^{15,16}$ in whom the symptomatology and nystagmus persist despite the reiteration of the maneuvers, and others in whom it recurs after the symptomatology has been solved (around 25\%, especially in the first 6 to 12 months).

In these cases, it is always necessary to ratify the diagnosis, excluding other causes of positional vertigo different from BPPV. ${ }^{9}$ The pathologies that can be confused with BPPV are grouped into three categories: otological, neurological and other entities. ${ }^{17}$ Among the otological pathologies are Ménière disease, unilateral acute vestibular syndrome (neuritis, labyrinthitis), superior semicircular canal dehiscence, dilated vestibular aqueduct, and perilymphatic fistula. ${ }^{17,18}$ The neurological causes include vestibular migraine, vestibular paroxysm, lesions in structures around the IV ventricle, cerebellar lesions, brainstem lesions (demyelinating lesions, tumors, and ischemic and degenerative lesions) and ArnoldChiari disease. We must bear in mind the association between BPPV and other vestibular pathologies such as Ménière disease, vestibular neuritis and migraine, so that, in the presence of a patient with specific symptoms, the possibility of presenting more than one vestibular disorder simultaneously should be considered. Other entities that can simulate BPPV are orthostatic hypotension, panic disorder or anxiety, drugs ${ }^{3}$ (primidone, carbamazepine, phenytoin, lithium, tranquilizers, antihypertensives), cervical rotational vertigo and positional convergence spasm. ${ }^{19}$

The objective of the following work is to assess the possible factors related to the persistence of BPPV and the importance of Magnetic Resonance Imaging (MRI) scans in the differential diagnosis of BPPV in cases that are refractory to the therapeutic maneuvers described.

\section{Methods}

A review of all cases of BPPV diagnosed in the Otorhinolaryngology Service of a tertiary hospital in the past 3 years with periodic follow-ups for a period of 6 months was performed. The canalicular repositioning maneuvers were always performed by the same otolaryngologists. A total of 176 patients were diagnosed and treated, and 150 of them were diagnosed with BPPV due to PSC canalolithiasis; 20 patients had BPPV from HSC; 10 of them had BPPV due to canalolithiasis; 10 of them had BPPV due to cupulolithiasis; 3 had BPPV due to SSC canalolithiasis; and 3 patients were diagnosed with PSC multicanal BPPV and HSC, for a total of 176 treated BPPVs. For the diagnosis, the high suspicion of BPPV was fundamentally based on the anamnesis. In addition, a complete otoneurologic exploration was performed, with otomicroscopy, acoumetry, exploration of spontaneous nystagmus in the five positions of the look and provoked nystagmuys by specific maneuvers. The Dix Hallpike (DH) maneuver was performed to explore the PSC and the SCC, while the McClure maneuver or Roll test was performed to explore the HSC. ${ }^{12}$ At the first examination, nystagmus was observed with the naked eyes (approximately half of the patients were diagnosed and initially treated at the Emergency Department). In all cases with difficult exploration of the nystagmus, doubts about its direction or poor response 
after one correctly executed maneuver, a videonistagmography was performed. We used the EM to treat the vertigo of the $\operatorname{SSC}(n=3)$ and PSC in all cases $(n=153)$, including those with involvement of more than one canal: a case of cupulolithiasis of the left HSC + canalolithiasis of the right PSC (the patient needed $4 \mathrm{Ems}$, and had subsequent instability), a case of cupulolithiasis of the right HSC + canalolithiasis of the right PSC (4 EMs and subsequent instability) and a case of canalolithiasis of the left HSC + canalolithiasis of the left PSC (1 EM). The "barbecue" manoeuver was used to treat the BPPV of the HSC, both for the cases of canalolithiasis and cupulolithiasis.

Within the refractory BPPV we included 3 categories: 1) positional vertigo with persistence of the nystagmus and of the symptomatology after 3 therapeutic maneuvers correctly performed; 2 ) those patients who maintained instability after the execution of 3 maneuvers, in spite of the negativization of the DH maneuver or of the Roll test; and 3) positional vertigo that initially responded to the maneuvers with resolution of the symptoms and the nystagmus, but recurred within a period of time shorter than 3 months. Finally, we divided the patients into two groups according to the response to the treatment: group A was composed of the cases refractory to the repositioning maneuvers, and group B was composed of the patients with a good response. We analyzed the otoneurologic background of every group to investigate a possible relation with the risk of refractory BPPV. We also considered the cases with affectation of more than one canal at the same time to evaluate if the presence of a multicanal BPPV was associated with a higher risk of refractory vertigo. The statistical analyses were performed by an independent statistician. For the comparisons between the two groups, odds ratio (OR) and Fisher exact test were used, and the statistical significance was defined at $p<0.05$. In all patients in whom it was necessary to repeat the maneuver on 3 or more occasions, we performed an imaging study through an MRI with gadolinium, to rule out other causes of BPPV, especially central ones.

\section{Results}

The mean age of the patients was 61.3 years, with a female preponderance and femaleto male ratio of 2.6:1 (128 females and 48 males, $72.8 \%$ and $27.2 \%$ respectively). With respect to the affected side, the right ear was more frequently affected (102 right ears and 77 left ears, also considering the patients with multicanal vertigo: right PSC + left HSC; right PSC + right HSC; left PSC + left HSC).

The efficacy of the EM at the first attempt reached 76.4\% (117 patients out of 153 with negative DH maneuver after an EM), and it was necessary to perform the EM twice in 18 patients (with negativization of the DH maneuver), 3 times in 11 patients (with negativization of the $\mathrm{DH}$ maneuver) and 4 times in 7 patients (with negativization of the DH maneuver in only 3 of them; in the remaining 4, Brandt-Daroff habituation exercises were required). At 6 months, negativization of the DH maneuver was achieved in $97.38 \%$ of all cases (149 out of of 153 patients). In 4 patients with persistent positive $\mathrm{DH}$ ( 2 of them with a history of vestibular neuritis and refractory to 4 maneuvers) and in 7 patients with persistent instability after 3 EMs but with negative DH (1 female patient with a history of stapedotomy in the 2 previous weeks), it was necessary to resort to the BrandtDaroff exercises and vestibular rehabilitation.

The BPPV of the HSC was treated with the "barbecue" maneuver described by Lempert, both for the cases of canalolithiasis and cupulolithiasis. Including the multicanal BPPV, we present 11 cases of canalolithiasis and 12 of cupulolithiasis. Of the 11 patients with canalolithiasis, in 8 (72.72\%) the Roll test was negativized, with the disappearance of the symptoms with a single maneuver. It was necessary to perform it twice in the other 3 patients $(27.28 \%)$. Of the 12 patients with cupulolithiasis, in 7 (58.33\%) 1 maneuver was sufficient; in 4 patients, 2 were performed; and, in 1 patient, the maneuver had to be performed 4 times. The rate of efficacy of the Lempert maneuver was of $72.72 \%$ in the cases of canalolithiasis, and of $58.33 \%$ in the cases of cupulolithiasis.

In the 3 patients who presented a multicanal BPPV, PSC and HSC, 1 canal was treated, and after solving it, the other was treated. They were a cupulolithiasis of the left HSC with canalolithiasis of the right PSC (4 EMs, 1 Semont maneuver and 2 Lempert maneuvers were performed); a right HSC cupulolithiasis with right PSC canalolithiasis (1 Lempert maneuver and 4 EMs were performed), and one left HSC canalolithiasis with left PSC canalolithiasis (1 Lempert maneuver and 1 EM were performed). ${ }^{12}$

In all of the cases that required three or more maneuvers, with complete resolution or not of the symptomatology, we requested a brain study with an MRI with gadolinium to rule out central causes of paroxysmal positional vertigo.

In total, MRIs were performed in 19 patients:

- Seven patients diagnosed with BPPV of posterior semicircular canal. Two suffered from multicanal vertigo: one case of left HSC cupulolithiasis + right PSC canalolithiasis and other case of right HSC cupulolithiasis + right PSC canalolithiasis. Out of these 7 , in 4 the DH maneuver was not negativized.

- Eleven patients diagnosed with BPPV of the PSC who needed 3 EMs, and in 3 of them the instability persisted after the maneuver.

- One patient diagnosed with BPPV due to cupulolithiasis of the right HSC, in which 4 Lempert maneuvers were performed, with persistence of the symptoms and nystagmus with the Roll test.

After 19 MRIs, a focal thickening of $2.4 \mathrm{~mm}$ of the right VIII cranial nerve $(\mathrm{CN})$ pair was diagnosed, and it was compatible with a small vestibular schwannoma in a 65-year-old female patient with BPPV due to cupulolithiasis of the right HSC refractory to 4 Lempert maneuvers (-Fig. 1 ).

Considering "normal" the realization of up to 3 canalicular repositioning maneuvers, out of a total of 19 that required 3 or more maneuvers, we actually considered 11 cases refractory ( $\mathbf{- T a b l e ~} \mathbf{1})$ :

- Seven patients with BPPV of the PSC who underwent 4 EMs, of which 3 did not negativize the DH maneuver (2 of them with a diagnosis of vestibular neuritis and 1 without 

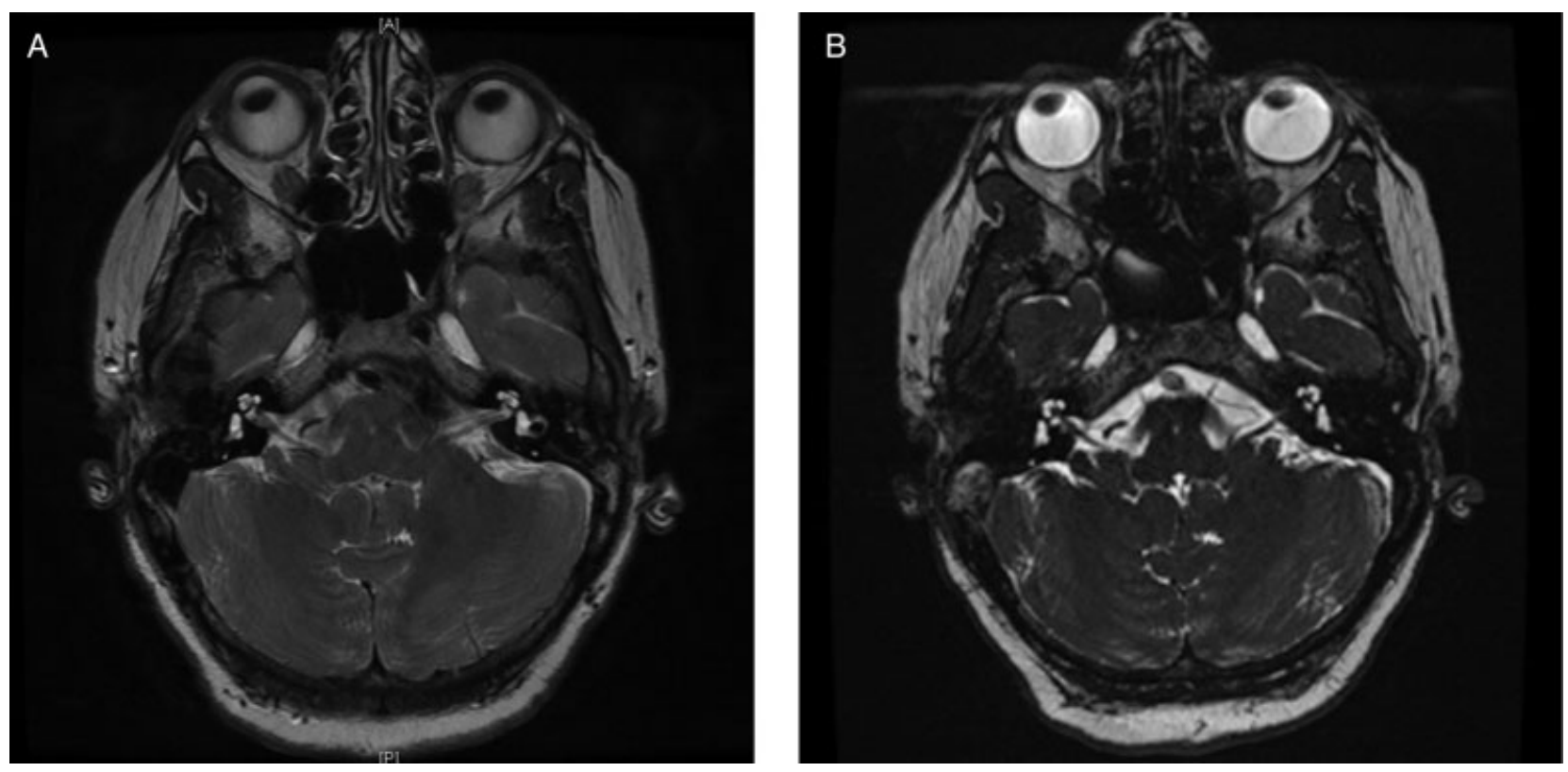

Fig. 1 (A) Magnetic resonance imaging scan (MRI) with gadolinium T2-weighted sequence; (B) MRI with gadolinium sequence FIESTA. The thickening of the VIII cranial nerve in the right internal auditory canal is observed.

a history). Of the 4 that negativized the DH maneuver, 2 were multiple-canal BPPVs and 2 were BPPVs without otoneurologic history.

- Three patients with BPPV of the PSC who maintained instability after 3 maneuvers ( 1 with a history of stapedotomy in the previous 15 days, and 2 without any history).

- One case of vertigo due to cupulolithiasis of the right HSC in which 4 Lempert maneuvers were performed (right vestibular schwannoma) without negativization of the Roll test and with persistence of the symptomatology.
A total of 3 out of these 11 patients presented otoneurologic background, 2 of them with history of vestibular neuritis (refractory to 4 maneuvers, without negativization of the DH maneuver), and 1 patient had undergone a stapedotomy two weeks before the onset of the symptoms (3 EMs with subsequent instability). Two other patients presented a multicanal vertigo, respectively a case of cupulolithiasis of the left HSC + canalolithiasis of the right PSC, and another one of cupulolithiasis of the right HSC with canalolithiasis of the right PSC (4 EMs and negativization of the DH maneuver). Finally, one patient was diagnosed with a

Table 1 Characteristics of the patients with refractory vertigo

\begin{tabular}{|l|l|l|l|l|l|}
\hline & Canal involved & Number of maneuvers & History & MRI & Resolution \\
\hline 1 & PSC & 4 Epley & Vestibular Neuritis & Negative & $\begin{array}{l}\text { DH positive; } \\
\text { Brandt-Daroff }\end{array}$ \\
\hline 2 & PSC & 4 Epley & Vestibular Neuritis & Negative & $\begin{array}{l}\text { DH positive; } \\
\text { Brandt-Daroff }\end{array}$ \\
\hline 3 & PSC & 4 Epley & None & Negative & $\begin{array}{l}\text { DH positive; } \\
\text { Brandt-Daroff }\end{array}$ \\
\hline 4 & PSC & 4 Epley & None & Negative & DH negative \\
\hline 5 & PSC & 4 Epley & None & Negative & $\begin{array}{l}\text { DH negative; } \\
\text { Roll test negative }\end{array}$ \\
\hline 6 & PSC + HSC & 4 Epley and 1 Lempert & None & Negative & $\begin{array}{l}\text { DH negative; } \\
\text { Roll Test negative }\end{array}$ \\
\hline 7 & $\begin{array}{l}\text { PSC }+ \text { HSC } \\
\text { cupulolithiasis }\end{array}$ & $\begin{array}{l}4 \text { Epley, 1 Semont } \\
\text { and 2 Lempert }\end{array}$ & None & Negative & Instability \\
\hline 8 & PSC & 3 Epley & Stapedo-tomy & Negative & Instability \\
\hline 9 & PSC & 3 Epley & None & Right VIII neurinoma & $\begin{array}{l}\text { Roll test positive; } \\
\text { vestibular rehabilitation }\end{array}$ \\
\hline 10 & PSC & 4 Lempert & None & & Instability \\
\hline 11 & HSC cupulolithiasis & & & & \\
\hline
\end{tabular}

Abbreviations: DH, Dix-Hallpike maneuver; HSC, horizontal semicircular canal; MRI, magnetic resonance imaging; PSC, posterior semicircular canal. 
right vestibular schwannoma associated with a cupulolithiasis of the right HSC refractory to 4 maneuvers.

The other 5 patients ( 1 without negativization of the $\mathrm{DH}$ maneuver after 4 EMs, 2 with negativization of the $\mathrm{DH}$ maneuver after 4 EMs, and 2 who maintained instability after 3 maneuvers) did not present a history of interest or other otoneurologic diagnosis.

In the 3 patients with instability after 3 EMs and in the 3 patients who did not negativize the $\mathrm{DH}$ maneuver after 4 EMs, the habituation treatment was proposed with BrandtDaroff exercises and vestibular rehabilitation, and all of them presented improvement in their symptoms.

The performance of an MRI with gadolinium was indicated in the cases that required the performance of 3 therapeutic maneuvers and the symptomatology was resolved (8/19). In none of them, the pathology was found through the MRI.

We divided the patients into two groups, refractory patients and good responders to the repositioning manoeuvers, and observed several differences.

Among the refractory patients (11/176-6.25\%), 4 of them (36.36\%) presented otoneurologic background or any otoneurologic pathology, and $2(18.18 \%)$ other patients were diagnosed with multicanal BPPV. We observed a female to male ratio of $9: 2$ (77.78\% and $22.22 \%$ respectively) and a mean age of 68.2 years. On the other hand, in the group of good responders (165/176-93.75\%), 15 patients presented otoneurologic background (9.09\%), only 1 was diagnosed with multicanal BPPV (0.60\%), the female to male ratio was 119:46 (61.35\% and 38.65\% respectively), and the mean age was 59.98 years (-Table $\mathbf{2}$ and - Table $\mathbf{3}$ ).

The statistical results showed that the presence of otoneurologic background was strongly associated with a higher risk of refractory BPPV (OR = 6.0952; 95\% confidence interval $[95 \% \mathrm{CI}]=1.5998-23.2234 ; p=0.0081$; Fisher exact test $=0.0199 ; 95 \% \mathrm{CI}$ ). When we analyzed every otoneurologic antecedent, we observed a statistically significant association between refractory vertigo and vestibular neuritis (OR $=18.1111 ; 95 \% \mathrm{CI}=2.2814-143.7740 ; p=0.0061$; Fisher exact test $=0.02 ; 95 \% \mathrm{CI}$ ). We also observed a statistically significant association between multicanal vertigo and a major risk of refractory vertigo $(\mathrm{OR}=36.4444 ; 95 \% \mathrm{CI}$ $=3.0140-440.6768 ; p=0.0047$; Fisher exact test $=0.0103$; 95\%CI). We didn't observe a statistically significant associa-
Table 3 Otoneurologic background of the two groups of patients

\begin{tabular}{|l|l|l|l|l|}
\hline $\begin{array}{l}\text { Otoneurologic } \\
\text { background }\end{array}$ & \multicolumn{2}{|l|}{$\begin{array}{l}\text { Group A } \\
(\boldsymbol{n}=11)\end{array}$} & \multicolumn{2}{l|}{$\begin{array}{l}\text { Group B } \\
(\boldsymbol{n}=165)\end{array}$} \\
\hline & $\mathbf{n}$ & $\%$ & $\mathbf{n}$ & $\%$ \\
\hline Vestibular neuritis & 2 & 18.18 & 2 & 1.21 \\
\hline Stapedectomy & 1 & 9.09 & 2 & 1.21 \\
\hline Vestibular schwannoma & 1 & 9.09 & & \\
\hline Ménière syndrome & & & 4 & 2.42 \\
\hline Sudden hearing loss & & & 2 & 1.21 \\
\hline Sudden tinnitus & & & 2 & 1.21 \\
\hline Labyrinthine fistula & & & 1 & 0.60 \\
\hline Vestibular migraine & & & 2 & 1.21 \\
\hline
\end{tabular}

tion between refractory vertigo and stapedectomy or vestibular schwannoma. Female sex and age were not associated with a higher risk of poor response to the treatment.

\section{Discussion}

Most cases of BPPV are idiopathic and respond successfully to canalicular repositioning maneuvers, and some even resolve without any treatment, being self-limiting episodes. ${ }^{15}$ Analyzing the results of previous studies, we observed that the failure rate of repositioning maneuvers varies between $4.5 \%$ and $32 \%$. In our series, the negativization of the DH maneuver was achieved in $97.38 \%$ of the cases after 3 maneuvers, with $2.62 \%(n=3)$ of patients continuing to have a positive DH and another $2.62 \%(n=3)$ with residual instability. All of them performed the BrandtDaroff habituation exercises and underwent vestibular rehabilitation with symptomatic improvement. In case of lack of response to 3 correctly performed maneuvers, or of frequent recurrence after initial improvement, other causes, especially neurological (central) and otological other than BPPV, should be ruled out. Neurological causes include vestibular migraine, vestibular paroxysm, lesions in structures around the IV ventricle, cerebellar lesions, brainstem lesions (demyelinating, tumor, ischemic and degenerative) and Arnold-Chiari disease. There is a series of warning signs in the exploration that may suggest the presence of

Table 2 Comparison of the two groups of patients

\begin{tabular}{|l|l|l|l|l|}
\hline & $\begin{array}{l}\text { Otoneurologic } \\
\text { background }\end{array}$ & Multicanal BPPV & F:M & Mean age \\
\hline $\begin{array}{l}\text { Group A (refractory vertigo; } \\
n=11 \text { ) }\end{array}$ & $4 / 11(36.36 \%)$ & $2 / 11(18.18 \%)$ & $9: 2(77.78 \%: 22.22 \%)$ & 68.2 years \\
\hline $\begin{array}{l}\text { Group B } \\
\text { good responders; } n=165)\end{array}$ & $15 / 165(9.09 \%)$ & $1 / 165(0.60 \%)$ & $119: 46(61.35 \%: 38.65 \%)$ & 59.8 years \\
\hline$p$-value (Fisher exact test, IC 95\%) & $p<0.05$ & $p<0.05$ & $p>0.05$ & $p>0.05$ \\
\hline
\end{tabular}

Abbreviations: BPPV, benign paroxysmal positional vertigo; F, female; M, male.

Notes: $p<0.05$ : statistically significant. Fisher exact test was used to compare the groups.

Otoneurologic background and multicanal affection were associated with refractory vertigo $(p<0.05)$. Sex and age didn't show any relation with refractory vertigo. 
paroxysmal positional vertigo of central origin, ${ }^{20}$ and that forces us to perform a MRI:

- Presence of signs or symptoms of CNS pathology (such as a gaze-evoked nystagmus).

- Absence of nystagmus inversion when inverting the position.

- Position-induced marked hyperemesis.

- A direction of the nystagmus that does not correspond to the duct that the maneuver explores, especially in the following cases: either persistent lower vertical positional nystagmus without torsional component or nystagmus that takes different directions in the diagnostic maneuvers performed repeatedly (provided that no replacement maneuvers have been performed, because, in this case, the particles could have moved from one conduit to another).

- Changing direction nystagmus without changes in the position of the head.

- Spontaneous nystagmus in the absence of positional maneuvers (discarding the positional pseudonystagmus observed in BPPV of HSC)

- Nystagmus without dizziness in the positional tests.

- Poor response to the therapeutic maneuvers.

- Multiple recurrences confirmed with diagnostic maneuvers.

Among the otological /otoneurologic diseases that can be confused with BPPV or that can be associated with it are chronic otitis media, Ménière syndrome, vestibular neuritis and perilymphatic fistula. ${ }^{21}$ The Ménière syndrome can begin with a BPPV-like symptomatology and be associated with BPPV, and, in this case, the BPPV is considered secondary.

Perez et $\mathrm{al}^{22}$ describe patients with Ménière syndrome in whom episodes of BPPV may precede, accompany or follow the classic outbreaks of Ménière syndrome. That is why a careful follow-up of the patients with BPPV is important.

Analyzing the series reported in the literature, in the series of 27 patients of Welling and Barness, ${ }^{23} 4$ cases of refractory BPPV were found, and the presence of a perilymphatic fistula after stapedotomy and a brain glioma were identified as causes of the failure of the maneuvers. In the series of 90 patients described by Rupa, ${ }^{15}$ out of the 7 refractory cases ( $\left.8 \%\right), 1$ was diagnosed with posterior fossa meningioma. In our series of 176 cases, among the 11 refractory patients (6.25\%), we identified a case of neurinoma of the right VIII CN. Although the causal relationship between BPPV and brain lesions is not known, a possible mechanism would be related to the vascular compromise of the labyrinth due to the ischemia of the anterior vestibular artery caused by the tumor. ${ }^{24}$

It is noteworthy that 4 out of the 11 cases considered refractory presented some otoneurologic background or otoneurologic pathology associated to BPPVs of more difficult control (2 cases of vestibular neuritis, 1 stapedectomy and 1 vestibular schwannoma), while only $15(9.09 \%)$ of the 165 patients with a good response presented a similar otoneurologic background. Although the number of patients is small, the results showed a statistically significant association between an otoneurologic background and refractory BPPV $(p<0.05)$, especially for vestibular neuritis. We also demonstrated that multicanal BPPV is more difficult to manage than simple BPPV, and it is associated with refractory vertigo, probably because it is more difficult to diagnose and requires special attention and knowledge of the pathophysiological patterns of positional vertigo. We must also bear in mind that the treatment of HSC is usually more complex, especially in the case of cupulolithiasis, and that BPPV of different canals sometimes coexist; therefore, when treating, for example, the PSC and not the HSC, the patient continues to present the symptoms. It is also possible to convert a BPPV of the PSC to a BPPV of the HSC, probably by passing the otoliths from the PSC to the HSC through the utricle. ${ }^{25}$ In our series we had three cases of multiple-canal BPPV with involvement of the PSC and HSC, and two of these required several maneuvers to be solved, with a statistically significant difference.

Finally, if a correct localization diagnosis has been made, identifying the semicircular canal involved and differentiating between canalolithiasis and cupulolithiasis, and if the specific maneuvers were not successful, we can use the Brandt-Daroff exercises, which, in some cases, have been shown to be more effective than the repositioning maneuvers. ${ }^{6}$

The present article has several methodological limitations. It is a retrospective study, so it is possible that bias occurred during the data collection. Since BPPV can precede, accompany or follow the classic symptoms of Ménière syndrome or vestibular neuritis, it is possible that several patients could be affected by an undiagnosed otoneurologic disease of which the BPPV was only the first manifestation. Another limitation is the small number of patients with a poor response, only 11 (6.25\%) cases versus $165(93.75 \%)$ of good responders, and this could influence the results observed in the study.

\section{Conclusions}

Although the EM, as well as the "barbecue" maneuver, if correctly performed, enable physicians to reach very high success rates, some patients are more difficult to treat, and an accurate differential diagnosis is mandatory. We observed that the presence of an otoneurologic background, especially vestibular neuritis and multicanal involvement, is statistically associated with cases of refractory BPPV, and we recommend performing an MRI with gadolinium to rule out vertigo of central origin. Despite our results, more research is needed to confirm the relationship between otoneurologic pathology and refractory BPPV.

\section{References}

1 Neira P, Valencuela V, Viada J. Semiótica del vértigo postural paroxístico benigno. Rev Otorrinolaringol Cir Cabeza Cuello 2001; 61:5-12

2 Froehling DA, Silverstein MD, Mohr DN, Beatty CW, Offord KP, Ballard DJ. Benign positional vertigo: incidence and prognosis in a population-based study in Olmsted County, Minnesota. Mayo Clin Proc 1991;66(06):596-601

3 Atacan E, Sennaroglu L, Genc A, Kaya S. Benign paroxysmal positional vertigo after stapedectomy. Laryngoscope 2001;111 (07):1257-1259

4 Rico-Romero BY, Ishiwara-Niembro JK, Sánchez-Pérez Y. Vértigo postural paroxístico benigno del canal semi-circular horizontal (BPPV-HSC). Rev Mex AMCAOF. 2012;1(02):119-125 
5 Cawthorne T. The physiological basis for head exercises. J Chart SocPhysiother. 1944;30:106-107

6 Steenerson RL, Cronin GW. Comparison of the canalith repositioning procedure and vestibular habituation training in forty patients with benign paroxysmal positional vertigo. Otolaryngol Head Neck Surg 1996;114(01):61-64

7 Brandt T, Daroff RB. Physical therapy for benign paroxysmal positional vertigo. Arch Otolaryngol 1980;106(08):484-485

8 Norré ME, Beckers A. Vestibular habituation training: exercise treatment for vertigo based upon the habituation effect. Otolaryngol Head Neck Surg 1989;101(01):14-19

9 Epley JM. Human experience with canalith repositioning maneuvers. Ann N Y Acad Sci 2001;942:179-191

10 Helminski JO, Zee DS, Janssen I, Hain TC. Effectiveness of particle repositioning maneuvers in the treatment of benign paroxysmal positional vertigo: a systematic review. Phys Ther 2010;90(05): 663-678

11 Dorigueto RS, Ganança MM, Ganança FF. The number of procedures required to eliminate positioning nystagmus in benign paroxysmal positional vertigo. Rev Bras Otorrinolaringol (Engl Ed) 2005;71(06):769-775

12 Carnevale C, Arancibia-Tagle DJ, Rizzo-Riera E, et al. Eficacia de las maniobras de reposicionamiento canalicular en el vértigo posicional paroxístico benigno: revisión de 176 casos tratados en un centro hospitalario de tercer nivel. Acta Otorrinolaringol Esp 2017;69(04):201-207

13 Brandt T, Huppert D, Hecht J, Karch C, Strupp M. Benign paroxysmal positioning vertigo: a long-term follow-up (6-17 years) of 125 patients. Acta Otolaryngol 2006;126(02):160-163

$14 \mathrm{Kim}$ JS, Oh SY, Lee SH, et al. Randomized clinical trial for geotropic horizontal canal benign paroxysmal positional vertigo. Neurology 2012;79(07):700-707
15 Rupa V. Persistent vertigo following particle repositioning maneuvers: an analysis of causes. Arch Otolaryngol Head Neck Surg 2004;130(04):436-439

16 Choi SJ, Lee JB, Lim HJ, et al. Clinical features of recurrent or persistent benign paroxysmal positional vertigo. Otolaryngol Head Neck Surg 2012;147(05):919-924

17 Bhattacharyya N, Baugh RF, Orvidas L, et al; American Academy of Otolaryngology-Head and Neck Surgery Foundation. Clinical practice guideline: benign paroxysmal positional vertigo. Otolaryngol Head Neck Surg 2008;139(05, Suppl 4):S47-S81

18 Parnes LS, Agrawal SK, Atlas J. Diagnosis and management of benign paroxysmal positional vertigo (BPPV). CMAJ 2003;169 (07):681-693

19 Huh YE, Kim JS. Bedside evaluation of dizzy patients. J Clin Neurol 2013;9(04):203-213

20 Soto-Varela A, Rossi-Izquierdo M, Sánchez-Sellero I, Santos-Pérez S. Revised criteria for suspicion of non-benign positional vertigo. QJM 2013;106(04):317-321

21 Honrubia V, Baloh RW, Harris MRJK, Jacobson KM. Paroxysmal positional vertigo syndrome. Am J Otol 1999;20(04):465-470

22 Perez N, Martin E, Zubieta JL, Romero MD, Garcia-Tapia R. Benign paroxysmal positional vertigo in patients with Ménière's disease treated with intratympanic gentamycin. Laryngoscope 2002;112 (06):1104-1109

23 Welling DB, Barnes DE. Particle repositioning maneuver for benign paroxysmal positional vertigo. Laryngoscope 1994;104 (8 Pt 1):946-949

24 Baloh RW, Honrubia V, Jacobson K. Benign positional vertigo: clinical and oculographic features in 240 cases. Neurology 1987; 37(03):371-378

25 Herdman SJ, Tusa RJ. Complications of the canalith repositioning procedure. Arch Otolaryngol Head Neck Surg 1996;122(03):281-286 